\title{
Motion Coordination of Planar Rigid Bodies
}

\author{
Rochelle Mellish and Derek A. Paley
}

\begin{abstract}
Motion coordination of autonomous vehicles has applications from target surveillance to climate monitoring. Previous research has yielded stabilizing control laws for a selfpropelled-particle model with first-order rotational dynamics, but this model may not adequately describe the rotational and translational dynamics of vehicles in the atmosphere or ocean. This paper describes the design of algorithms for decentralized control of self-propelled vehicles with second-order rotational and translational dynamics. We utilize a backstepping-based controller to achieve the desired second-order rotational dynamics of each vehicle and feedback linearization to attain the desired (constant) forward speed. We consider parallel and circular formations. These controls extend prior results to a more realistic vehicle model, while maintaining comparable performance.
\end{abstract}

\section{INTRODUCTION}

Dynamic models with ever increasing realism have been used to describe the cooperative behavior of agents working to accomplish a given task. In real-world systems the underlying dynamics unfold according to second-order differential equations, however many cooperative control schemes have been based on first-order models. In prior research, agents have been modeled as particles for simplicity and ease of control design. In some instances, the agents are modeled as unicycles, meaning that they can only move forward and turn. There are generally two control inputs in such models: a speed controller and a steering controller. Sometimes the problem is constrained further, as is the case when the translational speed of the platform is assumed to be constant. Examples of constant-speed models that rely solely on steering control are described in [11], [4], [8], [12], [1].

Implementing cooperative control laws based on particle dynamics enables only the location of the vehicle's center of mass to be controlled. Additional control inputs must be considered to ensure that not only the desired angular orientation is achieved, but also that the forward and transverse speeds achieve the desired values. Although there are numerous dynamic models that focus on the development of motion-planning algorithms for mobile robots, they tend to consider only a single platform rather than a swarm. One such example is [3], in which the motion-planning output of a kinematic model is used with a dynamic rigid body model in order to develop a trajectory for an autonomous vehicle

This material is based upon work supported by the National Science Foundation under Grant No. CMMI0928416 and the Office of Naval Research under Grant No. N00014-09-1-1058.

R. Mellish is a graduate student in the Department of Aerospace Engineering, University of Maryland College Park, College Park, MD 20742, USA rnmeumd. edu

D. Paley is an assistant professor in the Department of Aerospace Engineering, University of Maryland College Park, College Park, MD 20742, USA dpaleyeumd. edu that can avoid obstacles. Another example is [2], in which a Lagrangian formulation is used to derive the dynamic equations of a single mobile robot with non-holonomic constraints. To regulate movement of the robot, a velocity controller is used to generate torque control by way of integrator backstepping.

There is also a sizeable body of work that focuses on using kinematic models for each agent in a swarm, however, many such models typically do not incorporate rigid body dynamics. An example of a kinematic control for a multiagent system is [6], in which integrator backstepping is used for formation control of non-holonomic agents. A similar approach has been used in [7], in which the authors introduced a vehicle model based on [11] and [8] with secondorder rotational dynamics. We previously used integrator backstepping to adapt controllers designed for first-order rotational dynamics; however, forces and moments were not considered before now.

In this paper, we consider a collection of vehicles with second-order translational and rotational dynamics. We show that under existing second-order steering control laws provided in [7] and a new speed control, the planar rigid body model achieves comparable closed-loop performance to a particle model. In prior work, the vehicles were each considered to be particles with unit mass and unit speed and the direction of travel was controlled by a force orthogonal to the heading [8]. The vehicles we consider in the present paper can rotate and translate with variable speed subject to thrust, steering, and drag forces.

The contribution of this paper is to provide decentralized controllers for a collection of planar rigid bodies. These controllers enable the swarm to achieve parallel and circular formations using thrust and steering forces. We use feedback linearization to design the thrust controller and Lyapunovbased control to design the steering controller. We control the rigid-body dynamics to match those of a self-propelled particle; that is, each platform moves with constant speed in the desired formation.

This paper is organized as follows. Section II reviews the second-order particle model with steering controllers developed via backstepping. Section III describes the dynamics of a set of planar rigid bodies and Section IV derives the controllers used to create parallel and circular formations. Section V summarizes the paper and ongoing research. 


\section{BACKGROUND}

An existing model for planar collective motion with second-order rotational dynamics is

$$
\begin{aligned}
\dot{r}_{k} & =e^{i \theta_{k}} \\
\dot{\theta}_{k} & =\omega_{k} \\
\dot{\omega}_{k} & =a_{k},
\end{aligned}
$$

where $r_{k} \in \mathbb{C}$ is the position, $\theta_{k}$ is the heading, and $a_{k}$ represents the second-order steering control of vehicle $k=$ $1, \ldots, N$. In this model each vehicle moves at unit speed in the direction $\theta_{k}$. Model (1) represents an extension of a selfpropelled particle model with first-order rotational dynamics [7]. In the first-order case, phase and spacing potentials were used to derive control laws for parallel and circular formations in the absence of an external flowfield [11], and in the presence of a spatiotemporal flowfield [10]. Since firstorder rotational dynamics may not adequately describe rigidbody motion, these first-order particle models were used as the first component of a second-order system for which we designed a controller using integrator backstepping.

The expression for $a_{k}$ is found through the standard backstepping procedure to achieve $\omega_{k}=\phi_{k}$ as $t \rightarrow \infty$, where $\phi_{k}$ is the steering controller used to generate parallel and circular formations in the first-order system. We derive $a_{k}$ via the composite Lyapunov function

$$
V_{c}=V+\frac{1}{2} \sum_{k=1}^{N} z_{k}^{2},
$$

where $V$ is the smooth potential that must be minimized in order to achieve collective parallel or circular motion in the first-order system. The term $z_{k}=\omega_{k}-\phi_{k}$ is the error between the actual and desired first-order rotational dynamics. Taking the derivative of (2) along solutions of (1) gives

$$
\dot{V}_{c}=\sum_{k=1}^{N}\left[\frac{\partial V}{\partial \theta_{k}} \dot{\theta}_{k}+z_{k} \dot{z}_{k}\right],
$$

where $\dot{\theta}_{k}=\phi_{k}+z_{k}$ and $\dot{z}_{k}=v_{k}$ is a controller that we design to achieve $\dot{V}_{c} \leq 0$. The backstepping controller $a_{k}$ is found by the transformation

$$
a_{k}=v_{k}+\dot{\phi}_{k} .
$$

There are two types of motion primitives for which we derive $a_{k}$. The first is parallel formations, which has the key control parameter $p_{\theta}$ defined as [11]

$$
p_{\theta}=\frac{1}{N} \sum_{k=1}^{N} e^{i \theta_{k}} .
$$

$p_{\theta}$ represents the average linear momentum of the particles (assuming they have unit mass). Collective parallel motion is achieved by maximizing the average linear momentum of the particle system via the phase potential [11]

$$
V(\theta)=\frac{1}{2}\left(1-\left|p_{\theta}\right|^{2}\right) .
$$

For the first-order system, the controller for parallel formations $\dot{\theta}_{k}=\phi_{k}(\theta)$ is [11]

$$
\phi_{k}(\theta)=-K\left\langle p_{\theta}, i e^{i \theta_{k}}\right\rangle, K<0 .
$$

To achieve $\dot{V}_{c} \leq 0$ we choose

$$
v_{k}=\frac{1}{N}\left\langle p_{\theta}, i e^{i \theta_{k}}\right\rangle-\kappa z_{k},
$$

where $\kappa$ is a positive gain. This gives us

$$
\dot{V}_{c}=\sum_{k=1}^{N}\left[\frac{K}{N}\left\langle p_{\theta}, i e^{i \theta_{k}}\right\rangle^{2}-\kappa z_{k}^{2}\right] \leq 0 .
$$

The solutions $\theta_{k}$ and $z_{k}$ converge to the largest invariant set for which $\dot{V}_{c} \equiv 0$, given by

$$
\Lambda=\left\{\left\langle p_{\theta}, i e^{i \theta_{k}}\right\rangle \equiv 0, z_{k} \equiv 0 \forall k\right\} .
$$

The condition that $\left\langle p_{\theta}, i e^{i \theta_{k}}\right\rangle \equiv 0$ implies that $\theta_{j}=\theta_{k}$ for all particle pairs $j$ and $k$ [11]. $z_{k}=0$ implies $\omega_{k}=\phi_{k}(\theta)$; however, from the preceding condition we know that $\phi_{k}(\theta)=$ 0 in $\Lambda$. This implies that $\theta_{k}$ is constant for all $k$. Thus, $\Lambda$ contains the set of parallel formations.

Using transformation (4), a backstepping-based secondorder controller for parallel formations in (1) is [7]

$$
\begin{aligned}
a_{k}= & \frac{1}{N}\left\langle p_{\theta}, i e^{i \theta_{k}}\right\rangle-\kappa\left(\omega_{k}+K\left\langle p_{\theta}, i e^{i \theta_{k}}\right\rangle\right) \\
& -\frac{K}{N} \sum_{j=1}^{N}\left[\left\langle e^{i \theta_{j}}, e^{i \theta_{k}}\right\rangle\left(\omega_{j}-\omega_{k}\right)\right], K<0, \kappa>0 .
\end{aligned}
$$

The second motion primitive is circular formations. Collective circular motion is achieved by minimizing the spacing potential [11]

$$
V(\mathbf{r}, \theta)=\frac{1}{2}\langle\mathbf{c}, P \mathbf{c}\rangle .
$$

The $N \times 1$ matrix c contains the centers $c_{k}, k=1, \ldots, N$, of the circular paths followed by each particle, where [11]

$$
c_{k}=r_{k}+i \omega_{0}^{-1} e^{i \theta_{k}} .
$$

$P=I_{N \times N}-\frac{1}{N} \mathbf{1 1}^{T}$ is an $N \times N$ matrix that projects onto the space orthogonal to $\mathbf{1}=[1, \ldots, 1]^{T} \in \mathbb{R}^{N}$ and $P_{k}$ represents the $k$ th row of $P$. For the first-order system, the controller for circular formations is [11]

$$
\phi_{k}(\mathbf{r}, \theta)=\omega_{0}\left(1+K\left\langle P_{k} \mathbf{c}, e^{i \theta_{k}}\right\rangle\right), K>0 .
$$

To achieve $\dot{V}_{c} \leq 0$ we choose

$$
v_{k}=-\kappa z_{k}+\omega_{0}^{-1}\left\langle P_{k} \mathbf{c}, e^{i \theta_{k}}\right\rangle,
$$

which gives us

$$
\dot{V}_{c}=\sum_{k=1}^{N}\left[-K\left\langle P_{k} \mathbf{c}, e^{i \theta_{k}}\right\rangle^{2}-\kappa z_{k}^{2}\right] \leq 0 .
$$

The solutions $\theta_{k}$ and $z_{k}$ converge to the largest invariant set $\Lambda$ for which $\dot{V}_{c} \equiv 0$, given by

$$
\Lambda=\left\{\left\langle P_{k} \mathbf{c}, e^{i \theta_{k}}\right\rangle \equiv 0, z_{k} \equiv 0 \quad \forall k\right\} .
$$

$\left\langle P_{k} \mathbf{c}, e^{i \theta_{k}}\right\rangle=0$ implies that $P_{k} \mathbf{c}=0$, which is only true when all circular centers are the same; that is, $P_{k} \mathbf{c}=0$ if and only if $\mathbf{c}$ is in the span of $\mathbf{1} .\left\langle P_{k} \mathbf{c}, e^{i \theta_{k}}\right\rangle=z_{k}=0$; thus we have $\dot{\theta}_{k}=\phi_{k}(\theta)=\omega_{0}$. Thus, all $N$ particles travel around the same circle of radius $1 /\left|\omega_{0}\right|$.

Using transformation (4), a backstepping control to stabilize circular formations is [7]

$$
\begin{aligned}
a_{k}= & -\kappa\left(\omega_{k}-\phi_{k}(\mathbf{r}, \theta)\right)+\omega_{0} K \omega_{k}\left\langle\tilde{r}_{k}, i e^{i \theta_{k}}\right\rangle \\
& +K\left(\omega_{0}-\frac{1}{N} \sum_{j=1}^{N}\left[\left\langle e^{i \theta_{j}}, e^{i \theta_{k}}\right\rangle\left(\omega_{0}-\left(\omega_{j}-\omega_{k}\right)\right)\right]\right) \\
& +\omega_{0}^{-1}\left(\left\langle\tilde{r}_{k}, e^{i \theta_{k}}\right\rangle-\omega_{0}^{-1} \frac{1}{N} \sum_{j=1}^{N}\left\langle i e^{i \theta_{j}}, e^{i \theta_{k}}\right\rangle\right),
\end{aligned}
$$

where $\tilde{r}_{k}=r_{k}-\frac{1}{N} \sum_{j=1}^{N} r_{j}$, and $K>0, \kappa>0$. 
When a uniform, time-invariant flowfield is present, the second-order model (1) can be written as

$$
\begin{aligned}
\dot{r}_{k} & =e^{i \theta_{k}}+f_{k} \\
\dot{\theta}_{k} & =\omega_{k} \\
\dot{\omega}_{k} & =a_{k},
\end{aligned}
$$

where $f_{k} \in \mathbb{C}$ is the flowfield. This model can also be expressed more succinctly using the magnitude $s_{k}=\left|e^{i \theta_{k}}+f_{k}\right|$ and orientation $\gamma_{k}=\arg \left\{e^{i \theta_{k}}+f_{k}\right\}$ of the inertial velocity [8]:

$$
\begin{aligned}
\dot{r}_{k} & =s_{k} e^{i \gamma_{k}} \\
\dot{\gamma}_{k} & =\Omega_{k} \\
\dot{\Omega}_{k} & =\lambda_{k} .
\end{aligned}
$$

Similarly to the flow-free case, we may define the average linear momentum as $p_{\gamma}=\frac{1}{N} \sum_{k=1}^{N} e^{i \gamma}$. Thus, the controllers can be re-expressed as [7]

$$
\begin{aligned}
\lambda_{k}= & \frac{1}{N}\left\langle p_{\gamma}, i e^{i \gamma_{k}}\right\rangle-\kappa\left(\Omega_{k}+K\left\langle p_{\gamma}, i e^{i \gamma_{k}}\right\rangle\right) \\
& -\frac{K}{N} \sum_{j=1}^{N}\left[\left\langle e^{i \gamma_{j}}, e^{i \gamma_{k}}\right\rangle\left(\Omega_{j}-\Omega_{k}\right)\right]
\end{aligned}
$$

for parallel formations, and

$$
\begin{aligned}
\lambda_{k}= & -\kappa\left(\Omega_{k}-\phi_{k}(\gamma)\right)+\omega_{0} K \Omega_{k}\left\langle\tilde{r}_{k}, i e^{i \gamma_{k}}\right\rangle+\omega_{0} \dot{s}_{k} \\
& +K\left(\omega_{0} s_{k}-\frac{1}{N} \sum_{j=1}^{N}\left[\left\langle e^{i \gamma_{j}}, e^{i \gamma_{k}}\right\rangle\left(\omega_{0} s_{j}-\left(\Omega_{j}-\Omega_{k}\right)\right)\right]\right) \\
& +\omega_{0}^{-1}\left(\left\langle\tilde{r}_{k}, e^{i \gamma_{k}}\right\rangle-\omega_{0}^{-1} \frac{1}{N} \sum_{j=1}^{N}\left\langle i e^{i \gamma_{j}}, e^{i \gamma_{k}}\right\rangle\right)
\end{aligned}
$$

for circular formations. When we consider, without loss of generality, a flowfield oriented along the real axis given by $f=\alpha \in \mathbb{R}$, the particle speed is

$$
\dot{s}_{k}=-\alpha \sin \gamma_{k}\left[1+\frac{\alpha \cos \gamma_{k}}{\sqrt{1-\alpha^{2} \sin ^{2}\left(\gamma_{k}\right)}}\right] \Omega_{k} .
$$

While models (1) and (20) are useful for understanding the movement of particles in the plane, they may not be sufficient for describing rigid-body dynamics. The presence of secondorder rotational dynamics is only a part of the transformation process from particle model to rigid-body model. In order to complete the transformation, we consider second-order translational dynamics as well.

\section{PlanAR RIGID-BODY DYNAMICS}

Recall that in Eq. (1) each particle moves with unit forward speed and its direction of travel is controlled by $\theta_{k}$. We can alternately use $\theta_{k}$ to describe the orientation of a planar rigid body. With this description, $e^{i \theta_{k}}=\bar{x}_{k}$ defines the body-fixed frame $\mathscr{B}_{k}=\left(k, \bar{x}_{k}, \bar{y}_{k}, \bar{z}_{k}\right)$, where $\bar{z}_{k}$ is out of the plane and $\bar{y}_{k}=\bar{z}_{k} \times \bar{x}_{k}$.

Let $u_{k} \in \mathbb{R}$ represent the forward speed of the $k$ th vehicle in a body-fixed frame. The transverse speed in frame $\mathscr{B}_{k}$ is $v_{k} \in \mathbb{R}$. The $k$ th velocity expressed as components in $\mathscr{B}_{k}$ is

$$
\dot{r}_{k}=u_{k} \bar{x}_{k}+v_{k} \bar{y}_{k} .
$$

The time-derivative of (24) with respect to inertial frame $\mathscr{I}$ yields the following rigid-body translational kinematics:

$$
\begin{aligned}
\ddot{r}_{k} & =\dot{u}_{k} \bar{x}_{k}+u_{k} \dot{\theta}_{k} \bar{y}_{k}+\dot{v}_{k} \bar{y}_{k}-v_{k} \dot{\theta}_{k} \bar{x}_{k} \\
& =\left(\dot{u}_{k}-v_{k} \dot{\theta}_{k}\right) \bar{x}_{k}+\left(u_{k} \dot{\theta}_{k}+\dot{v}_{k}\right) \bar{y}_{k} .
\end{aligned}
$$

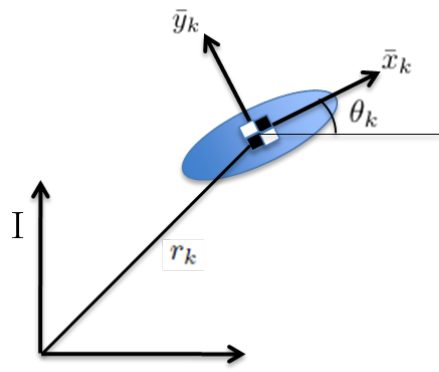

(a)

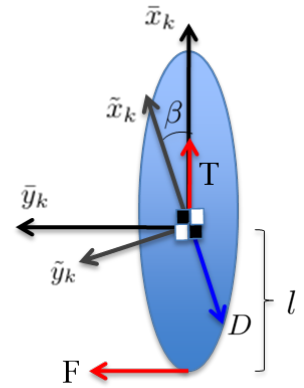

(b)
Fig. 1. (a) A single vehicle in the plane. (b) $\mathscr{B}_{k}$ and $\mathscr{C}_{k}$ reference frames; thrust $T_{k}$ and steering force $F_{k}$.

We assume there are two control forces acting on the $k$ th vehicle. The first is the thrust force $T_{k}$, which acts along the $\bar{x}_{k}$ axis. The steering control is $F_{k}$, which acts along the $\bar{y}_{k}$ axis a distance of $l$ away from the center of mass. We also include a drag force $D_{k}=\frac{1}{2} \rho s_{k}^{2} S C_{D}$. ( $\rho$ is the density of the medium through which the vehicle is traveling; $S$ is the vehicle reference area; and $C_{D}$ is the appropriate drag coefficient.) The vehicle speed is $s_{k}=\sqrt{u_{k}^{2}+v_{k}^{2}} \geq 0$.

We define a path frame for the $k$ th vehicle as $\mathscr{C}_{k}=$ $\left(k, \tilde{x}_{k}, \tilde{y}_{k}, \tilde{z}_{k}\right)$, where $\dot{r}_{k}=s_{k} \tilde{x}_{k}$ and $\tilde{z}_{k}=\bar{z}_{k}$. We denote the orientation of $\mathscr{C}_{k}$ relative to $\mathscr{B}_{k}$ as $\beta_{k}$. The drag force acts in the $-\tilde{x}_{k}$ direction [9]. Using Newton's second law with mass $m_{k}$ we have

$$
\begin{aligned}
& \left(T_{k}-D_{k} \cos \beta_{k}\right) \bar{x}_{k}+\left(F_{k}-D_{k} \sin \beta_{k}\right) \bar{y}_{k} \\
= & m_{k}\left(\dot{u}_{k}-v_{k} \dot{\theta}_{k}\right) \bar{x}_{k}+m_{k}\left(u_{k} \dot{\theta}_{k}+\dot{v}_{k}\right) \bar{y}_{k} .
\end{aligned}
$$

Collecting the $\bar{x}_{k}$ terms, we have

$$
T_{k}-D_{k} \cos \beta_{k}=m_{k}\left(\dot{u}_{k}-v_{k} \dot{\theta}_{k}\right) .
$$

We are able to obtain a dynamic expression for the forward speed $u_{k}$ by solving (27) for $\dot{u}_{k}$ :

$$
\dot{u}_{k}=-\frac{1}{m_{k}} D_{k} \cos \beta_{k}+\frac{1}{m_{k}} T_{k}+v_{k} \dot{\theta}_{k} .
$$

Following the same procedure for the terms in the $\bar{y}_{k}$ direction, we have

$$
\dot{v}_{k}=-\frac{1}{m_{k}} D_{k} \sin \beta_{k}+\frac{1}{m_{k}} F_{k}-u_{k} \dot{\theta}_{k}
$$

In order to design the rotational dynamics, let $M_{k}$ be the sum of the moments acting on the $k$ th vehicle. Assuming there is no moment due to drag (i.e, the drag acts through the vehicle's center of mass), we have

$$
M_{k}=\left(-l \bar{x}_{k}\right) \times\left(F_{k} \bar{y}_{k}\right)
$$

The rotational dynamics are

$$
I_{k} \ddot{\theta}_{k}=-l F_{k},
$$


where $I_{k}$ is the moment of inertia about the center of mass of vehicle $k$. The equations of motion are

$$
\begin{aligned}
& \dot{r}_{k}=u_{k} \bar{x}_{k}+v_{k} \bar{y}_{k}=s_{k} \tilde{x}_{k} \\
& \dot{u}_{k}=-\frac{1}{m_{k}} D_{k} \cos \beta_{k}+\frac{1}{m_{k}} T_{k}+v_{k} \omega_{k} \\
& \dot{v}_{k}=-\frac{1}{m_{k}} D_{k} \sin \beta_{k}+\frac{1}{m_{k}} F_{k}-u_{k} \omega_{k} \\
& \dot{\omega}_{k}=-\frac{l}{I_{k}} F_{k},
\end{aligned}
$$

where $\omega_{k}=\dot{\theta}_{k}, D_{k}=\frac{1}{2} \rho s_{k}^{2} S C_{D}$, and $\beta_{k}=\arccos \left(u_{k} / s_{k}\right)$.

\section{Stabilization of Moving Formations}

\section{A. Parallel Formation Control}

In this section we design a decentralized feedback control to drive a collection of planar rigid bodies in the same direction at the same speed. In order to ensure that the steady-state forward speed of each vehicle is $u_{k}=u_{0}$, we use feedback linearization [5]. Choosing

$$
T_{k}=m_{k}\left(\frac{1}{m_{k}} D_{k} \cos \beta_{k}-v_{k} \omega_{k}+K_{f}\left(u_{0}-u_{k}\right)\right),
$$

where $K_{f}>0$, yields the following closed-loop dynamics

$$
\dot{u}_{k}=K_{f}\left(u_{0}-u_{k}\right) .
$$

The dynamics (34) ensure $u_{k}$ exponentially converges to $u_{0}$.

Motivated by the backstepping procedure described in Section II, we choose

$$
F_{k}=-\frac{I_{k}}{l} a_{k},
$$

with $a_{k}$ given by (11).

Theorem 1: Consider the rigid body model (32) with thrust control (33) and steering control (35), where $a_{k}$ is given by (11). Under these controllers, the set of parallel formations where $\beta_{k}=0$ and $u_{k}=u_{0}$ for all $k$, and $\theta_{k}=\theta_{j}$ for all pairs $j$ and $k$ is asymptotically stable.

Proof: In order to analyze the closed-loop system, we propose the following candidate Lyapunov function

$$
U_{p a r}(\mathbf{u}, \mathbf{v}, \theta, \mathbf{z})=V_{c}+\frac{1}{2} \sum_{k=1}^{N}\left[\left(u_{0}-u_{k}\right)^{2}+v_{k}^{2}\right],
$$

where $V_{c}$ is given by (2) with phase potential (6). Taking the time-derivative along solutions of (32) gives

$$
\dot{U}_{\text {par }}=\dot{V}_{c}+\sum_{k=1}^{N}\left[-\left(u_{0}-u_{k}\right) \dot{u}_{k}+v_{k} \dot{v}_{k}\right] .
$$

Observe that $\dot{V}_{c} \leq 0$ under the steering control (35). Note $a_{k}=a_{k}(\theta)$ is independent of the states $u_{k}$ and $v_{k}$. If we plug in for $\dot{u}_{k}$ and $\dot{v}_{k}$ using (32) we obtain

$$
\begin{aligned}
\dot{U}_{p a r}= & \sum_{k=1}^{N}\left[\frac{K}{N}\left\langle p_{\theta}, i e^{i \theta_{k}}\right\rangle^{2}-\kappa z_{k}^{2}-K_{f}\left(u_{0}-u_{k}\right)^{2}\right. \\
& \left.-\frac{1}{m_{k}} v_{k} D_{k} \sin \beta_{k}+\frac{1}{m_{k}} v_{k} F_{k}-v_{k} u_{k} \omega_{k}\right] .
\end{aligned}
$$

The drag is $D_{k}=h s_{k}^{2} \geq 0$, where $h=\frac{1}{2} \rho S C_{D}$. Furthermore, $\sin \beta_{k}=v_{k} / s_{k}$, and we consider $s_{k} \geq 0$. Consequently, $-v_{k} D_{k} \sin \beta_{k}=-h s_{k} v_{k}^{2} \leq 0$.

According to the invariance principle, solutions converge to the largest invariant set $\Lambda$ in which $\dot{V}_{c}=0$, where $\Lambda$ is
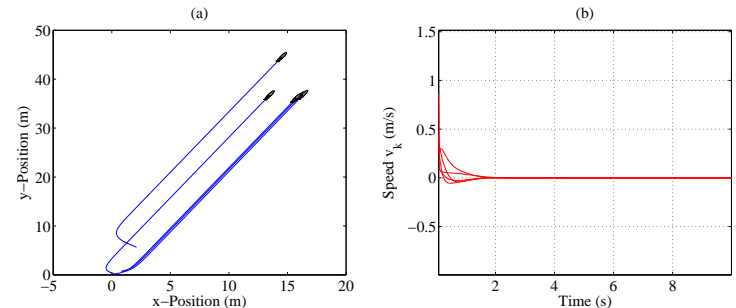

Fig. 2. (a) Rigid body parallel motion in the plane $[K=-1, \kappa=5$, and $u_{0}=1$ ]; (b) velocity component of parallel motion with time.

given by (10). In $\Lambda, \omega_{k} \equiv 0$ because $z_{k} \equiv 0$ and $\phi_{k} \equiv 0$. Note that $\omega_{k} \equiv 0$ only if $\dot{\omega}_{k}=0$ in $\Lambda$. Thus $F_{k} \equiv 0$ in $\Lambda$ because $\dot{\omega}_{k}=a_{k}=-\frac{I_{k}}{l} F_{k}$. In $\Lambda$,

$$
\dot{U}_{p a r}=\sum_{k=1}^{N}\left[-K_{f}\left(u_{0}-u_{k}\right)^{2}-\frac{h}{m_{k}} s_{k} v_{k}^{2}\right] \leq 0 .
$$

Application of the invariance principle in $\Lambda$ shows that solutions starting in $\Lambda$ converge to the largest set $M \subset \Lambda$ in which $\dot{U}_{p a r}=0$. In $M, u_{0}-u_{k} \equiv 0$ and $s_{k} v_{k}^{2} \equiv 0$ which implies $u_{k}=u_{0}$ and $v_{k}=0$. As a result, $\beta_{k} \equiv 0$ and $M$ contains the set of parallel formations. The remainder of the proof follows from [11, Theorem 1].

Theorem 1 is illustrated in Fig. 2.

\section{B. Circular Formation Control}

To stabilize circular formations, we use the thrust control (33). However, instead of using the steering control (35) with $a_{k}$ given by (18), we now consider an alternative backstepping control that takes into account the observation that for circular motion the steady-state crab angle $\beta_{k}$ is not zero. (For parallel motion, the steady-state crab angle is zero.)

To find the steady-state crab angle and the corresponding steady-state speed, we differentiate $\tan \beta_{k}=v_{k} / u_{k}$ with respect to time and solve for $\dot{\beta}_{k}$ with $u_{k}=u_{0} \neq 0$ to obtain

$$
\dot{\beta}_{k}=\frac{\cos ^{2} \beta_{k}}{u_{0}}\left(-\frac{1}{m_{k}} D_{k} \sin \beta_{k}+\frac{1}{m_{k}} F_{k}-u_{0} \omega_{k}\right) .
$$

For circular motion, $F_{k}=\dot{\omega}_{k}=0$, which implies

$$
\dot{\beta}_{k}=\frac{\cos ^{2} \beta_{k}}{u_{0}}\left(-\frac{1}{m_{k}} D_{k} \sin \beta_{k}-u_{0} \omega_{0} s_{k}\right) .
$$

The equilibrium points of (40) are

$$
\beta_{k}= \pm \pi / 2 \text { and } \beta_{k}=\arcsin \left(-\frac{m_{k} u_{0} \omega_{0}}{h s_{k}}\right),
$$

where we used $D_{k} \sin \beta_{k}=h s_{k} v_{k}$.

The equilibrium points $\beta_{k}= \pm \pi / 2$ are not possible for $u_{k}=u_{0} \neq 0$ since $\cos \beta_{k}=u_{k} / s_{k} \neq 0$. For the second set of equilibrium points, $v_{k}=s_{k} \sin \beta_{k}$ and $s_{k}$ are both constant. To see this, we solve

$$
s_{k}^{2}=u_{0}^{2}+\left(-\frac{m_{k} u_{0} \omega_{0}}{h}\right)^{2},
$$

obtaining

$$
s_{k}=\left(\frac{u_{0}^{2}+\sqrt{u_{0}^{4}+4\left(m_{k} u_{0} \omega_{0} / h\right)^{2}}}{2}\right)^{1 / 2} \triangleq s_{0}
$$


and

$$
v_{k}=-\frac{m_{k} u_{0} \omega_{0}}{h} \triangleq v_{0} .
$$

In light of this analysis, we modify the backstepping control (18) to allow for the vehicle speed $s_{k} \neq 1$. The first step is to recognize that, along solutions to (32), the circle center (13) becomes

$$
c_{k}=r_{k}+i \omega_{0}^{-1} e^{i\left(\theta_{k}+\beta_{k}\right)} .
$$

(We include the crab angle because the vehicle's total velocity heading must be tangent to the path.) The time derivative of (45) is $\dot{c}_{k}=\left(s_{k}-\omega_{0}^{-1}\left(\omega_{k}+\dot{\beta}_{k}\right)\right) e^{i\left(\theta_{k}+\beta_{k}\right)}$. As a result, the desired $\omega_{k}$ dynamics (14) become

$$
\phi_{k}(\mathbf{r}, \mathbf{u}, \mathbf{v}, \theta)=\omega_{0}\left(s_{k}+K\left\langle P_{k} \mathbf{c}, e^{i \psi_{k}}\right\rangle\right)-\dot{\beta}_{k}, K>0,
$$

where $\psi_{k}=\theta_{k}+\beta_{k}$. The backstepping control $\dot{\omega}_{k}=a_{k}$ that asymptotically stabilizes $\omega_{k}=\phi_{k}(\mathbf{r}, \mathbf{u}, \mathbf{v}, \theta)$ is

$$
\begin{aligned}
a_{k}= & -\kappa\left(\omega_{k}-\phi_{k}(\mathbf{r}, \mathbf{u}, \mathbf{v}, \psi)\right)+\omega_{0} K \dot{\psi}_{k}\left\langle\tilde{r}_{k}, i e^{i \psi_{k}}\right\rangle-\ddot{\beta}_{k} \\
+ & K\left(\omega_{0} s_{k}-\frac{1}{N} \sum_{j=1}^{N}\left[\left\langle e^{i \psi_{j}}, e^{i \psi_{k}}\right\rangle\left(\omega_{0} s_{j}-\left(\dot{\psi}_{j}-\dot{\psi}_{k}\right)\right)\right]\right) \\
& \omega_{0} \dot{s}_{k}+\omega_{0}^{-1}\left(\left\langle\tilde{r}_{k}, e^{i \psi_{k}}\right\rangle-\omega_{0}^{-1} \frac{1}{N} \sum_{j=1}^{N}\left\langle i e^{i \psi_{j}}, e^{i \psi_{k}}\right\rangle\right),
\end{aligned}
$$

where $\tilde{r}_{k}=r_{k}-\frac{1}{N} \sum_{j=1}^{N} r_{j}, K>0$, and $\kappa>0$. (In practice the $\omega_{0} \dot{s}_{k}, \dot{\beta}_{k}$, and $\ddot{\beta}_{k}$ terms are omitted, since they lead to a recursive formulation of the control and are zero in steady state.) We have the following result.

Theorem 2: Consider the rigid body model (32) with thrust control (33) and steering control (35), where $a_{k}$ is given by (47). Under these controllers, the set of circular formations with radius $1 /\left|\omega_{0}\right|$ and direction of rotation determined by the sign of $\omega_{0}$ is asymptotically stable. In this set, $u_{k}=u_{0}$ and $v_{k}=v_{0}$ for all $k$ and $c_{j}=c_{k}$ for all pairs $j$ and $k$, where $v_{0}$ is given in (44).

Proof: To determine if the chosen controllers for $T_{k}$ and $F_{k}$ establish the desired closed loop behavior, we begin by defining a Lyapunov function for circular formations as

$$
U_{\text {circ }}(\mathbf{r}, \mathbf{u}, \mathbf{v}, \psi, \mathbf{z})=V_{c}+\frac{1}{2} \sum_{k=1}^{N}\left[\left(u_{0}-u_{k}\right)^{2}+\left(v_{0}-v_{k}\right)^{2}\right]
$$

where $V_{c}$ is given by (2) with circular potential (12) and $c_{k}$ given by (45). Taking the time-derivative along solutions of (32) gives

$$
\begin{aligned}
\dot{U}_{\text {circ }}= & \sum_{k=1}^{N}\left[-K\left\langle P_{k} \mathbf{c}, e^{i \psi_{k}}\right\rangle^{2}-\kappa z_{k}^{2}\right. \\
& -\left(u_{0}-u_{k}\right)\left(-\frac{1}{m_{k}} D_{k} \cos \beta_{k}+\frac{1}{m_{k}} T_{k}+v_{k} \omega_{k}\right) \\
& \left.+\left(v_{0}-v_{k}\right)\left(\frac{1}{m_{k}} D_{k} \sin \beta_{k}-\frac{1}{m_{k}} F_{k}+u_{k} \omega_{k}\right)\right] .
\end{aligned}
$$

Choosing $T_{k}$ to be the stabilizing control (33) ensures that $u_{k}$ converges to $u_{0}$ according to the closed-loop dynamics (34). Furthermore, $\dot{V}_{c} \leq 0$ along solutions of (32). Therefore, solutions converge to the largest invariant set $\Lambda$ in which $u_{k}-u_{0} \equiv 0$ and $\dot{V}_{c} \equiv 0$, i.e.,

$$
\Lambda=\left\{\left\langle P_{k} \mathbf{c}, i e^{i \psi_{k}}\right\rangle \equiv 0, z_{k} \equiv 0, u_{k} \equiv u_{0}, \forall k\right\} .
$$
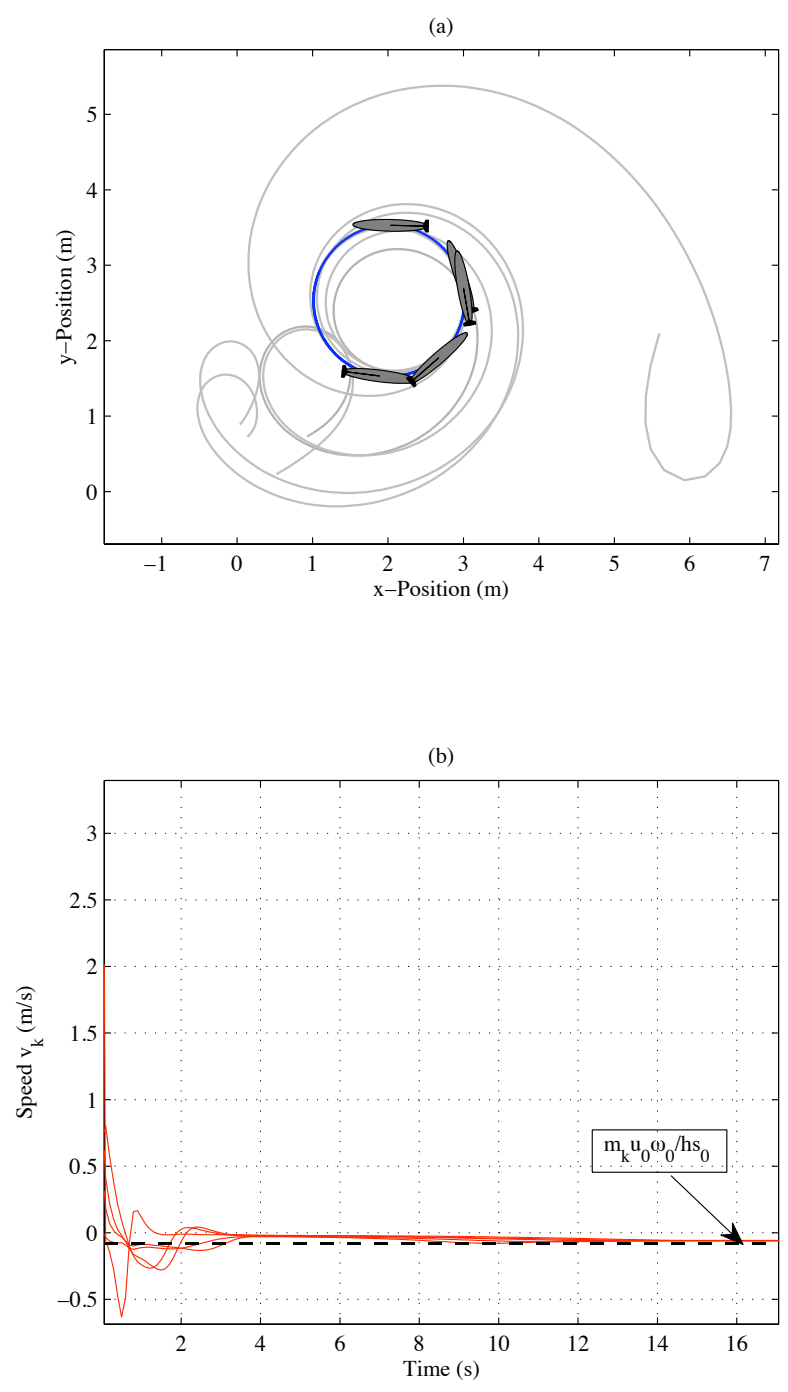

Fig. 3. (a) Rigid body circular motion in the plane $\left[K=1, \kappa=5, \omega_{0}=1\right.$, and $\left.u_{0}=1\right]$; (b) velocity components of circular motion with time.

In $\Lambda, \omega_{k} \equiv \omega_{0} s_{k}$ and $F_{k} \equiv 0$, which implies that $s_{k} \equiv s_{0}$. For solutions starting in $\Lambda$, we have

$$
\begin{aligned}
\dot{U}_{c i r c} & =\sum_{k=1}^{N}\left(v_{0}-v_{k}\right)\left(h s_{0} v_{k} / m_{k}+u_{k} \omega_{0} s_{0}\right) \\
& =\sum_{k=1}^{N}-\frac{h s_{0}}{m_{k}}\left(v_{0}-v_{k}\right)^{2} \leq 0 .
\end{aligned}
$$

Application of the invariance principle in $\Lambda$ shows that solutions starting in $\Lambda$ converge to the largest set $M \subset \Lambda$ in which $\dot{U}_{\text {circ }}=0 . M$ contains the set of circular formations with $u_{k} \equiv u_{0}$ and $v_{k} \equiv v_{0}$.

Theorem 2 is illustrated in Fig. 3.

\section{CONCLUSION}

In this paper we describe a planar rigid body model for motion coordination. In addition to forward dynamics in 
the body-fixed frame, second-order transverse dynamics are included in the model. The condition that the vehicle move at constant forward speed is relaxed; controllers are designed to ensure that the forward speed of each vehicle decays to the desired speed $u_{0}$. Decentralized controllers are provided to stabilize parallel and circular formations and illustrated in simulation. In ongoing work, we are extending these results to incorporate a steady, uniform flowfield.

\section{REFERENCES}

[1] N. Ceccarelli, M. Di Marco, A. Garulli, and A. Giannitrapani. Collective circular motion of multi-vehicle systems with sensory limitations. In Proceedings of the 44th IEEE Conference on Decision and Control, and the European Control Conference 2005, pages 740-745, Seville, Spain, December 2005.

[2] R. Fierro and F. L. Lewis. Control of a nonholonomic mobile robot: Backstepping kinematics into dynamics. In Proceedings of the 34th IEEE Conference on Decision and Control, pages 3805-3810, New Orleans, LA, December 1995.

[3] E. Frazzoli, M. A. Dahleh, and E. Feron. Real-time motion planning for agile autonomous vehicles. Journal of Guidance, Control, and Dynamics, 25(1):116-129, January - February 2002.

[4] E. W. Justh and P. S. Krishnaprasad. Equilibria and steering laws for planar formations. Systems and Control Letters, 52(1):25-38, May 2004.
[5] H. Khalil. Nonlinear Systems. Prentice Hall, Upper Saddle River, NJ 07458, 3rd. edition, 2002.

[6] K. D. Listmann and C. A. Woolsey. Output synchronization of systems in chained form. In Joint 48th IEEE Conference on Decision and Control and 28th Chinese Control Conference, pages 3341 - 3346, Shanghai, P.R. China, December 2009.

[7] R. Mellish, S. Napora, and D. A. Paley. Backstepping control design for motion coordination of self-propelled vehicles in a flowfield. International Journal of Robust and Nonlinear Control, 21(12):14521466, August 2011.

[8] D. A. Paley and C. Peterson. Stabilization of collective motion in a time-invariant flowfield. Journal of Guidance, Control, and Dynamics, 32(3):771-779, May-June 2009.

[9] D. A. Paley and D. Warshawsky. Reduced-order dynamic modeling and stabilizing control of a micro-helicopter. In Proceedings of the 47th AIAA Aerospace Sciences Meeting including the New Horizons Forum and Aerospace Exposition, Orlando, Florida, January 2009.

[10] C. Peterson and D. A. Paley. Multi-vehicle coordination in an estimated time-varying flowfield. AIAA Journal of Guidance, Control, and Dynamics, 34(1):177-191, 2011.

[11] R. Sepulchre, D. A. Paley, and N. Leonard. Stabilization of planar collective motion: All-to-all communication. IEEE Transactions on Automatic Control, 52(5):811-824, May 2007.

[12] R. Sepulchre, D. A. Paley, and N. E. Leonard. Stabilization of planar collective motion with limited communication. IEEE Transactions on Automatic Control, 53(3):706-719, April 2008. 